\title{
Prediction of Oral Bioavailability: Challenges and Strategies
}

\author{
Chunli Zheng and Yonghua Wang* \\ Center of Bioinformatics, College of Life Science, Northwest A \& F University, Yang ling, Shaanxi, China
}

\section{Editorial}

Oral Bioavailability $(\mathrm{OB})$ is defined as "the rate and extent to which the active ingredient or active moiety is absorbed from a drug product and becomes available at the site of action" by FDA [1]. For the pharmaceutical industries, high $\mathrm{OB}$ is often the major consideration in the development of bioactive molecules as therapeutic agents [2]. Meanwhile, for the drugs administrated by the oral route, OB is indisputably one of the most important pharmacokinetic parameters owing to its indicator role for the efficiency of the drug delivery to the systemic circulation [3]. Unfortunately, $\mathrm{OB}$ measured in vivo is labor intensive, time-consuming and prone to error. Therefore, in silico methods that can predict OB reliably and quickly are good alternatives. In this comment, an $\mathrm{OB}$ issue encountered during the course of prediction is described, including possible challenges and strategies.

\section{The Inherent Challenges in OB Prediction}

$\mathrm{OB}$ is governed by various biological and physicochemical properties, such as drug disintegration and dissolution, degradation, gastric emptying, the intestinal membrane permeation, the intestinal and hepatic first-pass metabolism, human intestinal absorption and even the drug dosage form. Worse still, the factors discussed above may differ across patients, and even during different periods of the same patient. Whether a drug is taken with or without food/other drugs may affect absorption and first-pass metabolism. Moreover, disease states play a role in affecting liver metabolism or gastrointestinal function and thus alter absorption.

\section{The Brief History of OB Prediction}

In recent years, various efforts have been reported to model OB using the descriptor-based quantitative-structure activity relationship approach (QSAR) methods, but their prediction abilities were relatively low. The major defects are high false positives rate, small sample sizes, and access protected.

The pioneers of $\mathrm{OB}$ prediction can be traced back to the regression model developed by Andrews and coworkers [4]. This model might not be reliable for its high false positives rate. Subsequently, the classification models were proposed [5]. However, the data set was smaller, and thus the reliability of them is questionable. In addition, Turner et al. [6] constructed a stepwise regression model with a correlation of 0.72 for the test set. But obviously, the test sets used in the model for validation was small which might also not be statistically reliable. Then, many models based on larger databases were proposed. Veber et al. [2] reported a QSAR model using 1100 drug candidates. Further, a QSPR model [7] for human absolute OB was proposed. These two models have something in common- data sources and access protected.

More recently, researchers are turning their attentions to develop new algorithm-aided prediction model. Ma et al. [8] proposed a GACG-SVM based prediction model with receivable overall classification accuracy, but unreasonable prediction accuracy for the lowbioavailability class. In 2011, Tian et al. [3] constructed MLR models by employing the genetic function approximation (GFA) technique. However, the prediction ability of the model is relatively low.

\section{Strategies and Perspective}

It is repeatedly specified that the perfect prediction model for OB should be physiologically and sufficiently reflective of the specific biological barrier of interest in humans [9]. This emphasizes that the knowledge of the metabolism and efflux at the intestinal mucosal level is of particular importance. In our previous work, we have constructed a novel chemometric method [10] for prediction of human OB by integrating the information of the ATP-dependent efflux protein $\mathrm{P}$-gp and the cytochrome P450s, the important defense limiting the absorption of candidate drugs. The optimal SVR model performs well with $\mathrm{R}^{2}$ of 0.80 and SEE of 0.31 for test sets, indicating the potential efficiency in early elimination of unfavorable candidates in drug discovery. But, there are still some limitations in this model, such as the lack of consideration of drug dosage form, disease states and so forth.

Developing a perfect prediction model will be challenging, but it is justified given their power to health care. The world cannot afford to wait long for answers to exact $\mathrm{OB}$, and researches need to play their part in finding solutions. For example, they will need to measure the influence of different dosage forms for OB. More broadly, drug interactions, food, disease states, gene polymorphism, individual medicine and many other factors should be taken into considerations. In addition, at this critical time, it would encourage industry and academia to promote cooperation, and become activists for $\mathrm{OB}$ prediction.

\section{Acknowledgements}

The research is supported by the Fund of Northwest A \& F University and is financially supported by the National Natural Science Foundation of China (Grant No. 31170796 and 81373892 ).

\section{References}

1. Barnes PM, Bloom B, Nahin RL (2008) Complementary and alternative medicine use among adults and children: United States, 2007. Natl Health Stat Report : 1-23.

2. Gahche J et al. (2011) Dietary supplement use among U.S. adults has increased since NHANES III (1988-1994). NCHS Data Brief 61: 1-8.

3. Briefel RR, Johnson CL (2004) Secular trends in dietary intake in the United States. Annu Rev Nutr 24: 401-431.

4. Eisenberg DM, Davis RB, Ettner SL, Appel S, Wilkey S, et al. (1998) Trends in alternative medicine use in the United States, 1990-1997: results of a follow-up national survey. JAMA 280: 1569-1575.

5. Sparreboom A, Cox MC, Acharya MR, Figg WD (2004) Herbal remedies in the United States: potential adverse interactions with anticancer agents. J Clin Oncol 22: 2489-2503.

${ }^{*}$ Corresponding author: Yonghua Wang, Center of Bioinformatics, College of Life Science, Northwest A \& F University, Yang ling, Shaanxi, China, Tel \& Fax: +86-029-87092262; E-mail: yh_wang@nwsuaf.edu.cn

Received December 17, 2013; Accepted December 20, 2013; Published December 28, 2013

Citation: Zheng C, Wang Y (2013) Prediction of Oral Bioavailability: Challenges and Strategies. J Bioequiv Availab 6: e47. doi:10.4172/jbb.10000e47

Copyright: (c) 2013 Zheng C, et al. This is an open-access article distributed under the terms of the Creative Commons Attribution License, which permits unrestricted use, distribution, and reproduction in any medium, provided the original author and source are credited. 
6. Gorman GS, Coward L, Darby A, Rasberry B (2013) Effects of herbal supplements on the bioactivation of chemotherapeutic agents. J Pharm Pharmacol 65: 1014-1025.

7. Ullmann U, Haller J, Decourt JP, Girault N, Girault J, et al. (2003) A single ascending dose study of epigallocatechin gallate in healthy volunteers. J Int Med Res 31: 88-101.

8. Chow HH, Hakim IA, Vining DR, Crowell JA, Ranger-Moore J, et al. (2005) Effects of dosing condition on the oral bioavailability of green tea catechins after single-dose administration of Polyphenon $E$ in healthy individuals. Clin Cancer Res 11: 4627-4633.

9. Zhou L, Zuo Z, Chow MS (2005) Danshen: an overview of its chemistry pharmacology, pharmacokinetics, and clinical use. J Clin Pharmacol 45: 13451359.

10. Wu B, Liu M, Zhang S (2007) Dan Shen agents for acute ischaemic stroke Cochrane Database of Systematic Reviews.

11. Cheng TO (2007) Cardiovascular effects of Danshen. Int J Cardiol 121: 9-22. 